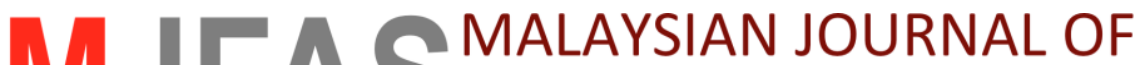 FUNDAMENTAL AND APPLIED SCIENCES PRINT ISSN: 2289-5981 | ONLINE ISSN: 2289-599X
}

\section{First-principles calculations of antimony sulphide $\mathrm{Sb}_{2} \mathrm{~S}_{3}$}

\author{
Afiq Radzwan a, Rashid Ahmed a,", Amiruddin Shaari a, Abdullahi Lawal a,b, Ying Xuan Ng a \\ a Department of Physics, Faculty of Science, Universiti Teknologi Malaysia, UTM Johor Bahru, Johor, Malaysia \\ b Department of Physics, Federal College of Education, Zaria, Nigeria \\ * Corresponding author: rashidahmed@utm.my
}

Article history

Received 18 February 2017

Accepted 9 August 2017

\begin{abstract}
The structural, electronic, as well as the optical properties of the Sb2S3, are investigated using fullpotential (FP) linearized augmented plane wave (LAPW) method framed within density functional theory (DFT) via treating the exchange-correlation potential with Engel-Vosko generalized gradient approximation (EV-GGA). Electronic properties calculations were performed with and without taking into account the effects of spin-orbit coupling (SOC). From our results, we found that structural properties, the density of states and band structure are in good agreement with experimental results. The effects of SOC on electronic properties were found to be negligible. The calculated optical properties, namely, imaginary and real parts of the dielectric function, reflectivity, absorption coefficient, refractive index, extinction coefficient and energy loss function and their analysis showed; $\mathrm{Sb}_{2} \mathrm{~S}_{3}$ metal chalcogenide is a promising material for solar cells.
\end{abstract}

Keywords: Density Functional Theory, LAPW, Antimony Sulphide, Spin-orbit coupling, Electronic properties, Optical properties

(C) 2017 Penerbit UTM Press. All rights reserved

\section{INTRODUCTION}

Metal chalcogenides materials such as cadmium telluride (CdTe) and copper indium gallium selenide (CIGS) have been known as an excellent candidate for high-performance photovoltaic devices like a solar cell. Though metal chalcogenides materials are highly efficient, the high cost, as well as high toxicity, limit their applications [1,2]. Therefore, low-cost, abundant and less toxic materials are needed [3]. A recent study showed that antimony sulphide $\left(\mathrm{Sb}_{2} \mathrm{~S}_{3}\right)$ hold great potential for photovoltaic application, due to its band gap having value in the range 1.65 to $2.4 \mathrm{eV}$ and high absorption coefficient in the visible region [4], obtained through a change in structure dimension [5-6]. $\mathrm{Sb}_{2} \mathrm{~S}_{3}$ semiconductor material belongs to metal chalcogenides group which is cheaper, abundant, and less toxic than any other compound in the group. Therefore, detailed knowledge of electronic and optical properties is essential for understanding its optoelectronic behavior such as band gap and light absorption. The band gap of a material determines its electrical conductivity [7] and optical properties that show the interaction between matter and electromagnetic radiation [8].

The role of the computational methods has been exploited to give an accurate prediction of the material properties in the numerous cases [9]. Several computational works have been reported on $\mathrm{Sb}_{2} \mathrm{~S}_{3}$ using different exchange-correlation functionals for getting reliable band structure and band gap value, but none of them included the effect of spin-orbit coupling (SOC). As spin-orbit interaction effect in heavy elements is substantial, therefore inclusion is important [10-12]. In this paper, with the inclusion of SOC, the calculation of band structure, the density of state (DOS), partial density of states (PDOS) and optical properties are computed by employing FP-LAPW method [13], realized in WIEN2k code [14]. The optical properties such as refractive index, extinction coefficient, absorption coefficient, energy-loss, and reflectivity obtained from the calculation of dielectric function are found in better consistency with the reported experimental results.

\section{COMPUTATIONAL METHOD}

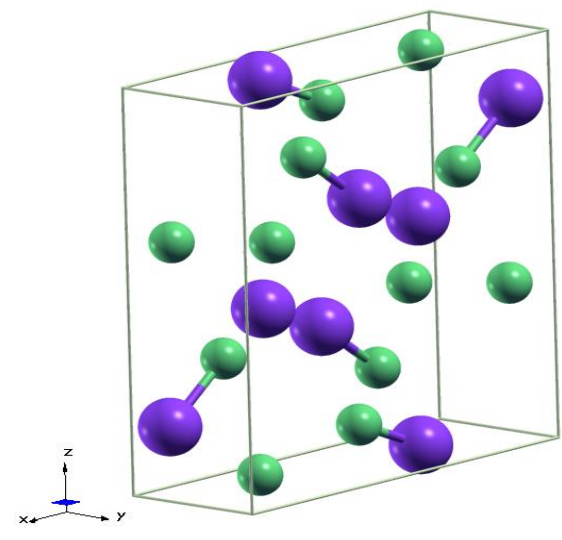

Fig. 1 Crystal structure of $\mathrm{Sb}_{2} \mathrm{~S}_{3}$

To compute the structural, electronic as well as the optical properties of $\mathrm{Sb}_{2} \mathrm{~S}_{3}$ in the orthorhombic crystal structure, FP-LAPW method as realized in WIEN2k code is used along with the inclusion of fully relativistic and SOC effects. The simulated $\mathrm{Sb}_{2} \mathrm{~S}_{3}$ crystallized structure in the orthorhombic crystal unit cell belonging to the spacegroup Pnma (62) is shown in Fig. 1. The initial structure parameters (lattice parameters and atomic positions) are used from the experimental study of Kyono and Kimata [15]. The electron exchangecorrelation functional was treated by the Engel Vosko generalized gradient approximation (EV-GGA) [16]. The muffin tin (MT) spheres radii of 2.0 and 2.43 a.u were chosen for $\mathrm{S}$ and $\mathrm{Sb}$, respectively. The convergence parameter $\mathrm{RK}_{\max }$ was set to be 9.0 shown in Eq. 1 for controlling the size of the basis set. The value of another parameter, $\mathrm{G}_{\max }$ in Eq. 2 was set to 12, where $\mathrm{G}_{\max }$ is taken as the largest vector for 
the Fourier expansion of the charge density [17]. To perform selfconsistent integration over the Brillouin zone (BZ), tetrahedron method was used with $28 \mathrm{k}$-points in the irreducible $\mathrm{BZ}$ corresponding to 250 k-points in the whole BZ. The self-consistent calculations were considered to be converged when the total energy was stabilized within $0.00001 \mathrm{Ry}$. For optical properties, a uniformly distributed denser kpoint mesh of 1000 were used.

$$
\begin{gathered}
\Psi=\sum_{K_{n}}^{K M A X} c_{K_{n}} e^{i K_{n} r} \\
\rho(r)=\sum_{G}^{G M A X} \rho_{G} e^{i G r}
\end{gathered}
$$

\section{RESULTS AND DISCUSSION}

\section{Structural optimization}

Investigation of the structural properties is the first step in DFT calculations to avoid a certain error for accurate predictions of other quantities. The lattice parameter $a, b$ and $c$ of $\mathrm{Sb}_{2} \mathrm{~S}_{3}$ in orthorhombic structure were determined via energy optimization and is shown along with other previous experimental and theoretical values as can be seen in Table 1. Our calculated results reasonably comparable with the experimental measurments.

\section{Electronic Structure}

The band structure calculation of the $\mathrm{Sb}_{2} \mathrm{~S}_{3}$ along high-symmetry points of the first Brillouin zone are plotted as shown in Fig. 2. The direct gap of $\mathrm{Sb}_{2} \mathrm{~S}_{3}$ with and without $\mathrm{SOC}$ is of $1.64 \mathrm{eV}$ and $1.60 \mathrm{eV}$ respectively. Table 2 represents a summary of the band gap results obtained in this work along with available theoretical and experimental data from other researchers [4-5]. The obtained band gap with SOC is very close to experimental value [5] compared with other previous DFT calculations. Similarly, our results with EV-GGA approximation are much better than that the results obtained at the level of generalized gradient approximation by Perdew, Burke and. Ernzerhof (PBE-GGA) functional. However, our results are lower than the reported experimental measurement [6], this may be due to the limitation of the first-principles approach based on DFT [18] or experimental measurements have some issue as well because compare to the PBEGGA, GGA functional constructed by Engel and Vosko usually show improvement in reproducing the band gap closer to the experimental band gap. The SOC normally reduces the band gap. Our obtained results with SOC are in line as reported by Pourghazi et. al for chalcogenide compound [19]. The results of total densities of states
(DOS) helps to further elaborate the nature of band structure whereas the partial density of states (PDOS) gives information about the origin of bands. Our calculated DOS and partial DOS for $\mathrm{Sb}_{2} \mathrm{~S}_{3}$ with the Fermi energy level represented by a reference line at zero energy is shown in Fig. 3. The results of DOS reveals that p-orbitals of Sb and S are the main contributors to the occupied states.

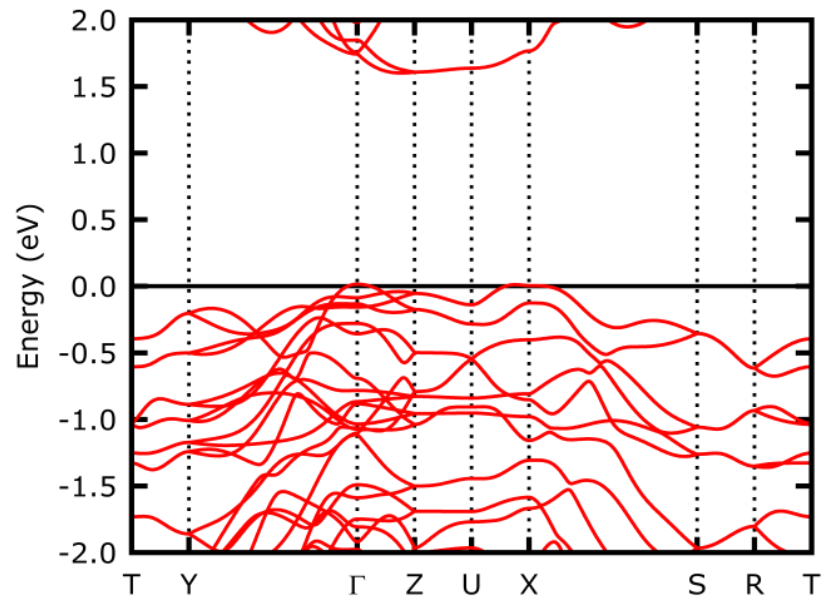

(a)

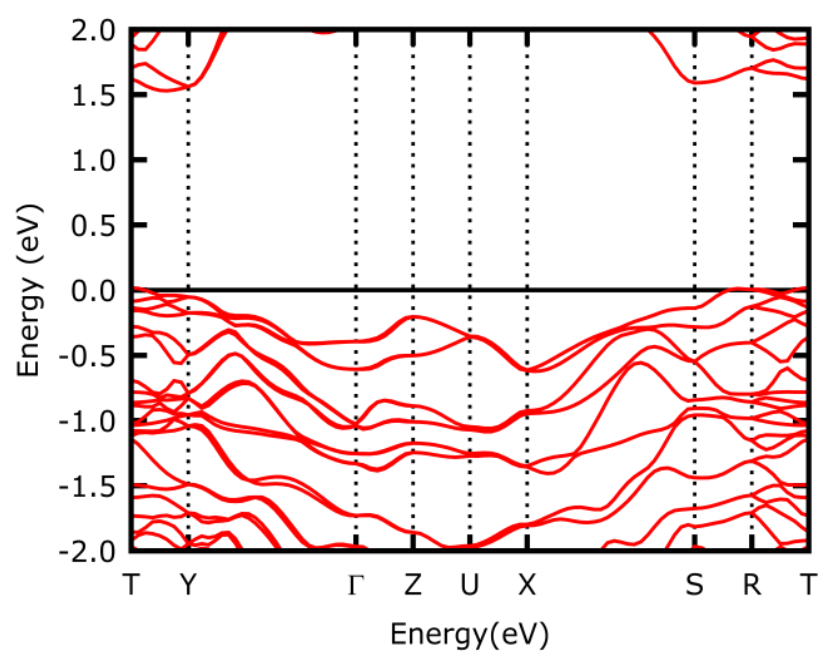

(b)

Fig. 2 Bandstructure of Sb2S3 without (a) and with SOC (b)

Table 1 Calculated equilibrium lattice constants of $\mathrm{Sb}_{2} \mathrm{~S}_{3}$

\begin{tabular}{cccc}
\hline Ref. & $\boldsymbol{a}(\AA)$ & $\boldsymbol{b}(\AA)$ & $\boldsymbol{c}(\AA)$ \\
\hline This work & 11.646 & 3.953 & 11.587 \\
Exp [15] & 11.311 & 3.839 & 11.223 \\
\hline
\end{tabular}

Table 2 Calculated and experimental energy gaps of $\mathrm{Sb}_{2} \mathrm{~S}_{3}$.

\begin{tabular}{cc}
\hline Band gap energy $(\mathrm{eV})$ & Ref. \\
\hline 1.64 & EV-GGA \\
1.60 & EV-GGA (SOC) \\
1.20 & PBE-GGA [20] \\
1.65 & Exp [5] \\
2.24 & Exp [6] \\
\hline
\end{tabular}




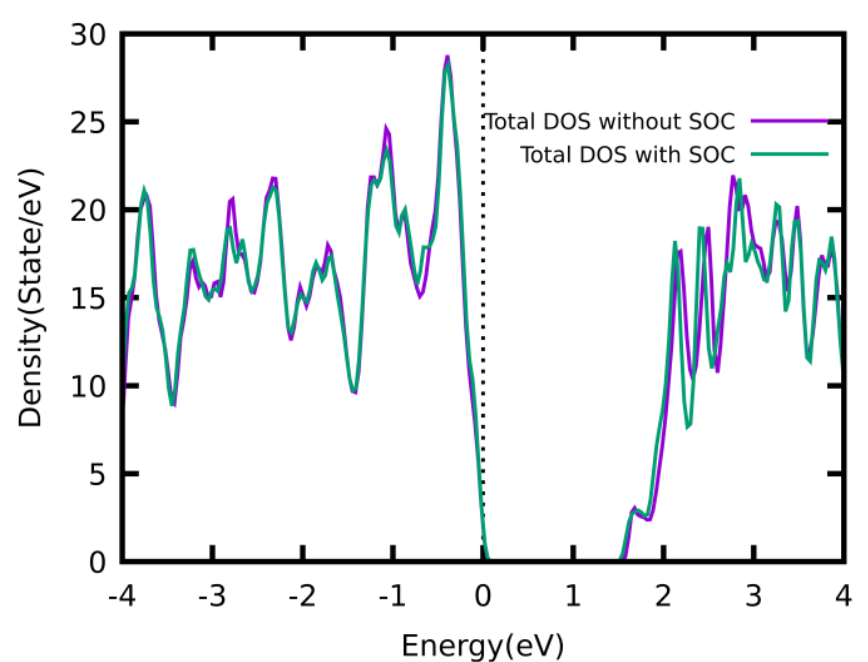

(a)

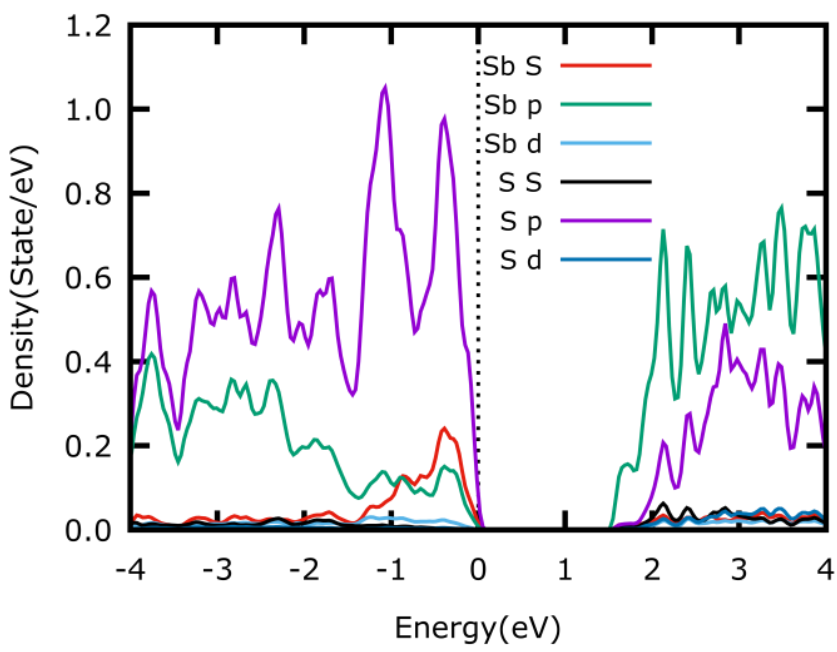

(b)

Fig. 3 Calculated total density of states (DOS) with and without SOC (a) partial density of states (PDOS) of $\mathrm{Sb}_{2} \mathrm{~S}_{3}$ for $\mathrm{Sb}$ and $\mathrm{S}$ with $\mathrm{SOC}(\mathrm{b})$

\section{Optical properties}

Investigation of the optical properties of materials is essential for understanding their optoelectronic nature, this can be achieved through linear response behaviour with electromagnetic radiation. The electronic and ionic contributions to the real $\varepsilon_{1}(\omega)$ and imaginary $\varepsilon_{2}(\omega)$ parts of frequency dependent dielectric function, $\varepsilon(\omega)=\varepsilon_{1}(\omega)+\varepsilon_{2}(\omega)$ as a function of incident photon can be determined from the band structure calculation via Wien2k code with the help of Kramers-Kronig relations [21]. With the knowledge of frequency dependent dielectric function, optical quantities, namely, absorption coefficient $\alpha(\omega)$, energy loss function $L(\omega)$, refractive index $n(\omega)$, extinction coefficient $k(\omega)$ and reflectivity $R(\omega)$ can be obtained as can be seen in the following expressions:

$\varepsilon_{1}(\omega)=1+\left(\frac{2}{\pi}\right) \int_{0}^{\infty} \mathrm{d} \omega^{\prime} \frac{\omega^{\prime 2} \varepsilon_{2}\left(\omega^{\prime}\right)}{\omega^{\prime 2}-\omega^{2}}$

$n=\sqrt{\left(\frac{\sqrt{\varepsilon_{1}^{2}(\omega)+\varepsilon_{2}^{2}(\omega)}+\varepsilon_{1}(\omega)}{2}\right)}$

$k=\sqrt{\frac{\sqrt{\varepsilon_{1}^{2}(\omega)+\varepsilon_{2}^{2}(\omega)}-\varepsilon_{1}(\omega)}{2}}$

$$
\begin{aligned}
& \alpha(\omega)=\frac{\omega}{c} \sqrt{2\left(\sqrt{\varepsilon_{1}^{2}(\omega)+\varepsilon_{2}^{2}(\omega)}-\varepsilon_{1}(\omega)\right)} \\
& L(\omega)=\frac{\varepsilon_{2}(\omega)}{\varepsilon_{2}(\omega)+\varepsilon_{1}(\omega)} \\
& R(\omega)=\left|\frac{\sqrt{\varepsilon(\omega)}-1}{\sqrt{\varepsilon(\omega)}+1}\right|^{2}
\end{aligned}
$$

A single crystal of $\mathrm{Sb}_{2} \mathrm{~S}_{3}$ is the optically trixial system because it has orthorhombic structure crystal structure. Therefore, its linear dielectric tensor of the $\mathrm{Sb}_{2} \mathrm{~S}_{3}$ have three independent components. As shown in the Fig. 4(a) any of the directions has different value for dielectric function constant. The electronic at high frequency and ionic contributions of a non-polar system is contained in the static dielectric permittivity tensor $\varepsilon(0)$. The dielectric constant $\varepsilon(\infty)$ at high frequency obtained in $\mathrm{x}, \mathrm{y}$ and $\mathrm{z}$ direction are 9.29434, 131.547, and 126.892, respectively. In the imaginary part of the dielectric function, the onset of the absorption edge $\varepsilon_{2}(\omega)$ called optical absorption edge occurred at $1.61 \mathrm{eV}$ for $\mathrm{x}, \mathrm{y}$ and $\mathrm{z}$ direction, which is due to the inter-band transition between conduction band minimum and valence band maximum states as can be seen in Fig. 4 (b). This value is corresponds to fundamental band gap shown in the Table 2. Moreover spectrum shape and optical gap value is found in quite good agreement with experimental results as well[4].

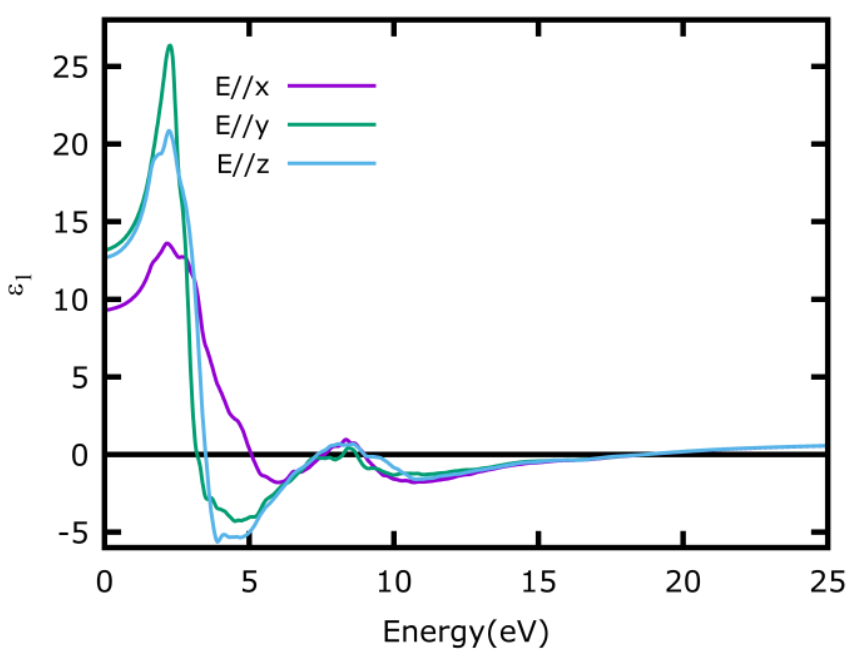

(a)

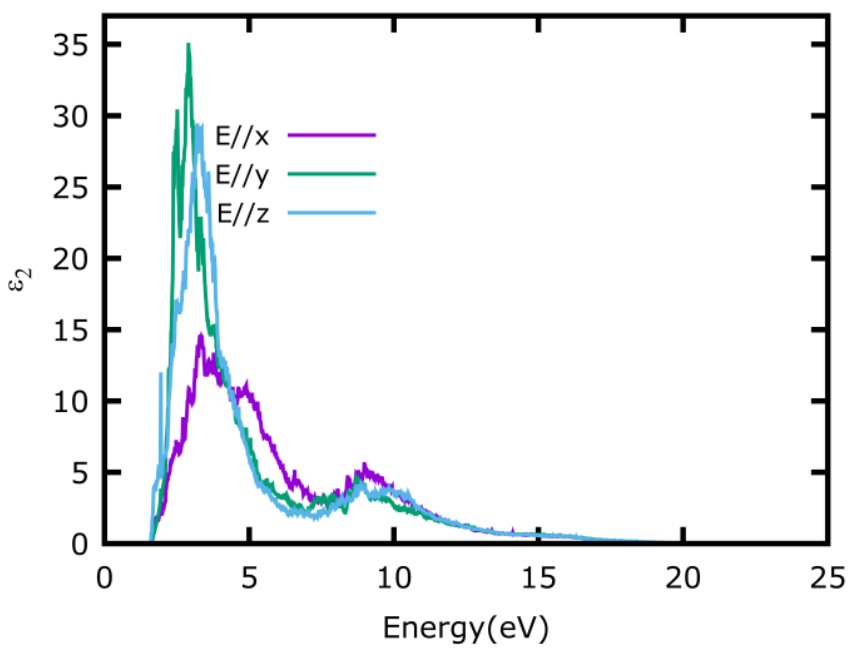

(b)

Fig 4 The calculated (a) real $\varepsilon_{1}(\omega)$ and (b) imaginary $\varepsilon_{2}(\omega)$ parts of the complex dielectric constant of $\mathrm{Sb}_{2} \mathrm{~S}_{3}$ 
The energy dependence absorption coefficient $\alpha(\omega)$, refractive index $\mathrm{n}(\omega)$, extinction coefficient $\mathrm{k}(\omega)$, energy-loss $\mathrm{L}(\omega)$, and reflectivity $\mathrm{R}(\omega)$ are given in Fig. 5 (a)-(e). These results are derived from the calculations of the $\varepsilon(\omega)$. From the Fig. 5 (a), the absorption coefficient for $\mathrm{x}, \mathrm{y}, \mathrm{z}$-direction are $172.674 \mathrm{~cm}^{-1}, 152.248 \mathrm{~cm}^{-1}$ and $167.867 \mathrm{~cm}^{-1}$ respectively. Absorption coefficient along y-direction is close to the experimental measurement of $139.76 \mathrm{~cm}^{-1}$. Fig. 5(b) displays the changes of refractive index with photon energy. As shown in Fig. 5 (b), the calculated $\mathrm{n}(0)$ in $\mathrm{x}, \mathrm{y}, \mathrm{z}$-direction are found to be $3.054,3.638$ and 3.566 respectively. The average value of $\mathrm{n}(0)$ was found to be 3.29 which is higher as compared to the previously reported results, 2.087 [22], this may be due to the corresponding somewhat different DFT calculations. We observed that extinction coefficient $\mathrm{k}(\omega)$ and imaginary part of dielectric function $\varepsilon_{2}(\omega)$ have a similar

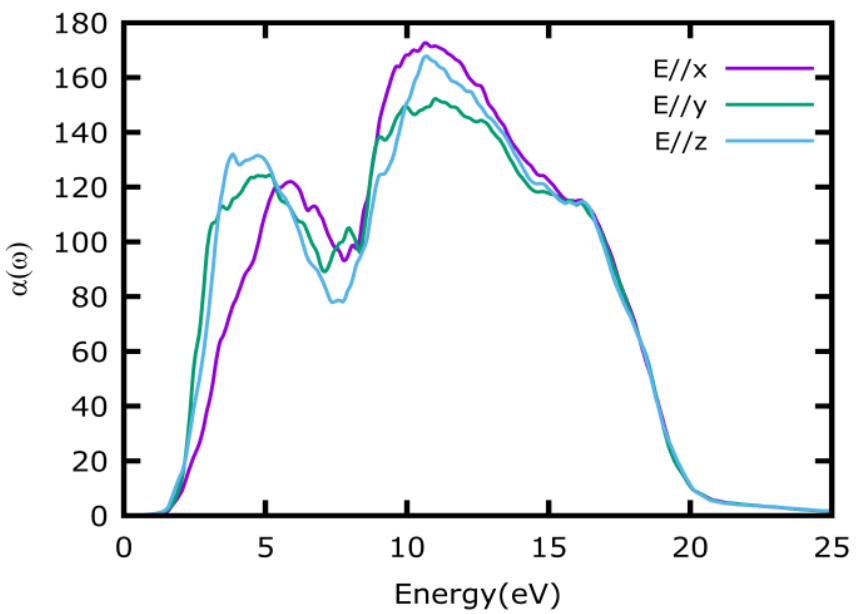

(a)

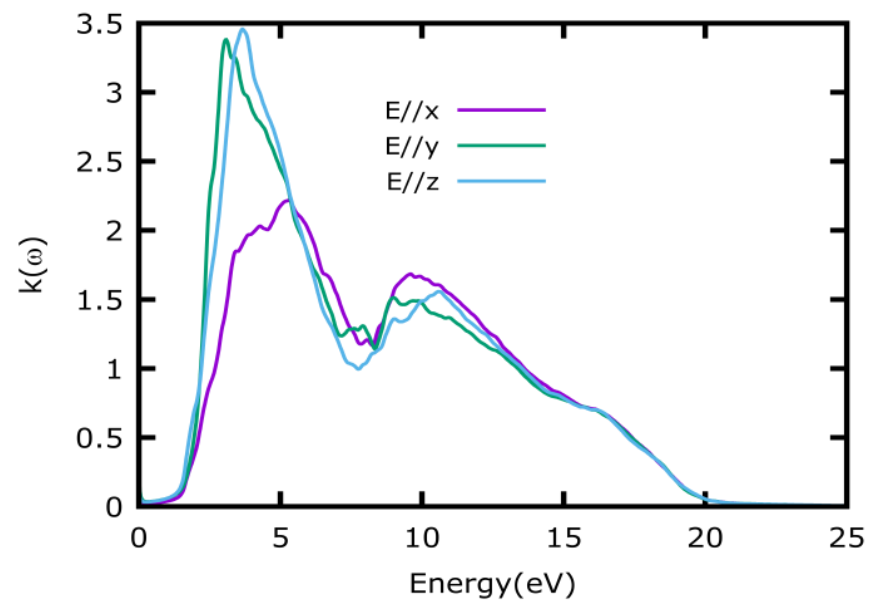

(c) trend as can be seen in Fig 5(c). Fig. 5 (d) is a graph of electron energy loss function $\mathrm{L}(\omega)$ with respect to the photon energy, this function describes energy loss of a fast moving electron through a material while moving from the top of a valence band to the bottom of the conduction band. The observed sharp maxima in the graph of the $\mathrm{L}(\omega)$ can be linked to the existence of plasma oscillations [23]. The resonant energy loss is seen at $18.898 \mathrm{eV}, 18.898 \mathrm{eV}$ and $18.844 \mathrm{eV}$ in $\mathrm{x}, \mathrm{y}$, and $\mathrm{z}$ directions respectively. Optical reflectivity is calculated and shown in Fig. 5(e). The main peaks are roughly at $5.728 \mathrm{eV}, 3.415 \mathrm{eV}, 3.850 \mathrm{eV}$. From the figure, it can be seen that the reflectivity is high in the visible light region $(1.65-3.1 \mathrm{eV})$ of the electromagnetic spectrum which makes the $\mathrm{Sb}_{2} \mathrm{~S}_{3}$ compound a good material for photovoltaic applications.

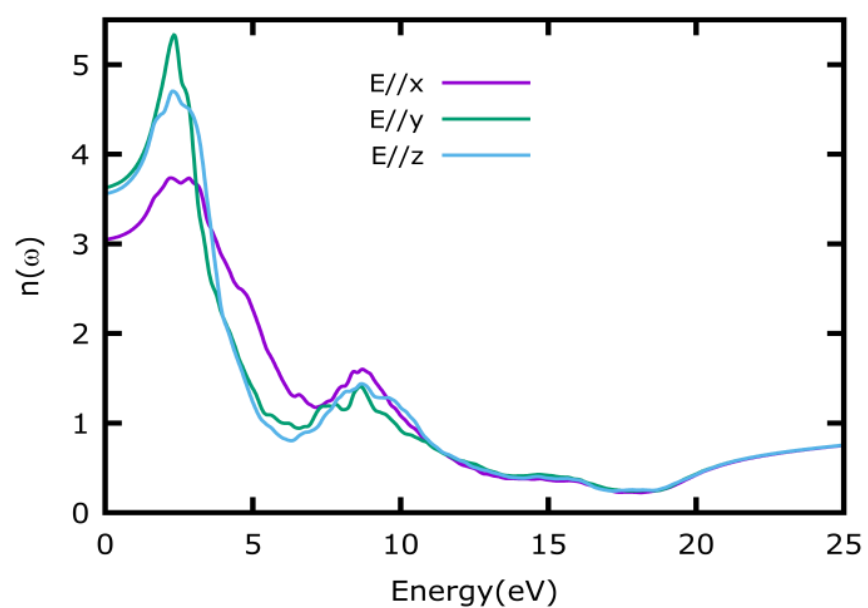

(b)

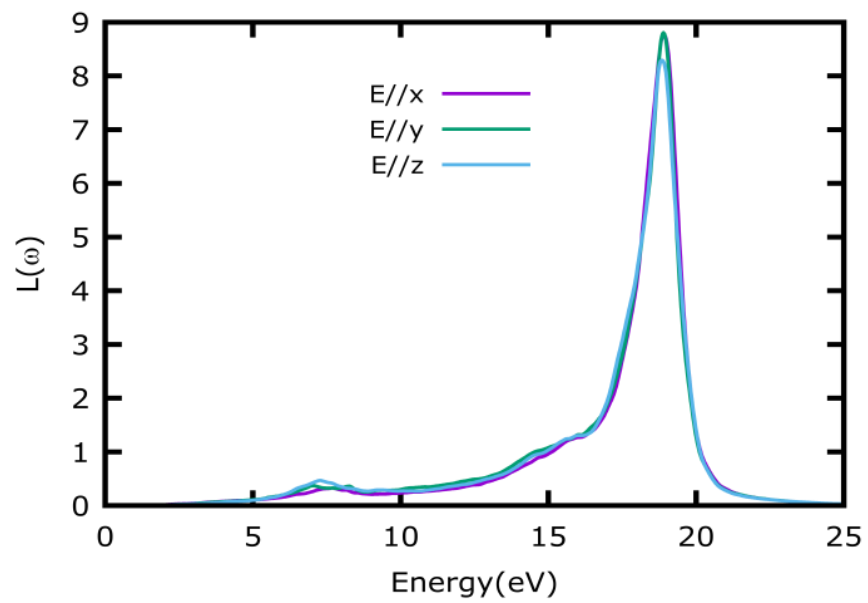

(d)

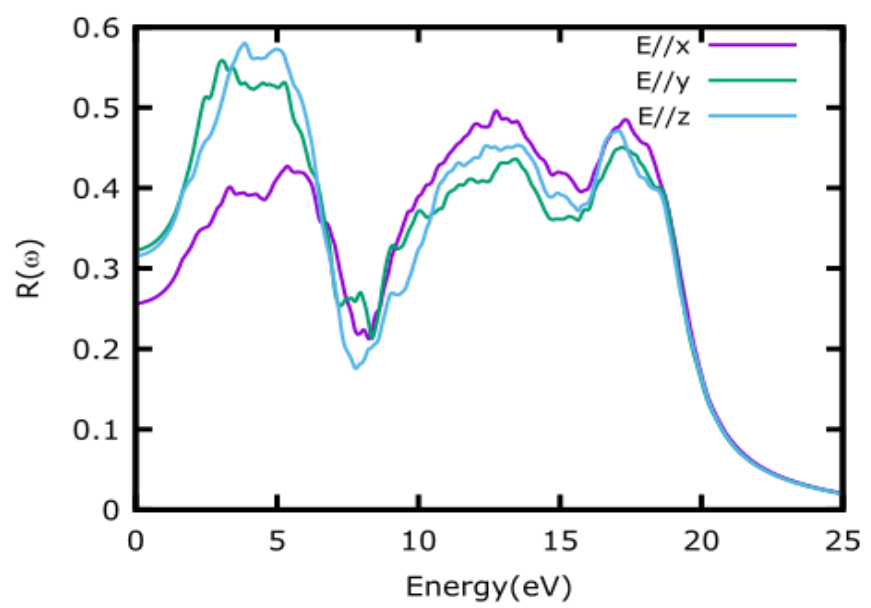

(e)

Fig. 5. Calculated optical constants of $\mathrm{Sb}_{2} \mathrm{~S}_{3}$ : absorption coefficient (a), refractive index (b), extinction coefficient (c), energy-loss (d) and reflectivity (e). 


\section{CONCLUSION}

In summary, the structural, electronic and optical properties of the $\mathrm{Sb}_{2} \mathrm{~S}_{3}$ crystalline compound were calculated using the FP-LAPW approach at the level of the EV-GGA exchange-correlation functional. The calculated lattice parameters were found closer to the experimental measurements. The fully relativistic effect within spin-orbit coupling (SOC) was included in the calculations of the electronic and optical properties. The calculated band structure provides an excellent description of the semiconducting behavior of $\mathrm{Sb}_{2} \mathrm{~S}_{3}$ with the band gap obtained the value $(1.60 \mathrm{eV})$ in close agreement with experimental measurements. Projected density of states reveals that the p-orbitals of $\mathrm{Sb}$ and $\mathrm{S}$ atoms are major contributors for conduction band. Optical properties calculations indicate that the optical gap is almost the same as energy band gap from the bandstructure calculation. Optical gap of $1.61 \mathrm{eV}$ shows that $\mathrm{Sb}_{2} \mathrm{~S}_{3}$ is a promising material for solar cell application.

\section{ACKNOWLEDGEMENT}

This work was financially supported by the Universiti Teknologi Malaysia under the Research University Grant (Vot No.: 12H46) and Ministry of Higher Education Malaysia.

\section{REFERENCES}

[1] Candelise, C., Winskel, M. \& Gross, R. (2012). Implications for CdTe and CIGS technologies production costs of indium and tellurium scarcity. Progress in Photovoltaics: Research and Applications, 20(6), 816-831.

[2] Razykov, T. M., Ferekides, C. S., Morel, D., Stefanakos, E., Ullal, H. S. \& Upadhyaya, H. M. (2011). Solar photovoltaic electricity: Current status and future prospects. Solar Energy, 85(8), 1580-1608.

[3] Todorov, T., Gunawan, O., Chey, S. J., De Monsabert, T. G., Prabhakar, A. \& Mitzi, D. B. (2011). Progress towards marketable earth-abundant chalcogenide solar cells. Thin Solid Films, 519(21), 7378-7381

[4] Chang, J. A., Rhee, J. H., Im, S. H., Lee, Y. H., Kim, H. J., Seok, S. I., Nazeeruddin, M. K. \& Gratzel, M. (2010). High-performance nanostructured inorganic-organic heterojunction solar cells. Nano Letters, 10(7), 2609-2612.

[5] Ali, N., Hussain, A., Ahmed, R., Shamsuri, W. N. W., Shaari, A., Ahmad, N., \& Abbas, S. M. (2016). Antimony sulphide, an absorber layer for solar cell application. Applied Physics A: Materials Science and Processing, 122(1), 1-7.
[6] García, R. A., Avendaño, C. M., Pal, M., Delgado, F. P. \& Mathews, N. R. (2016). Antimony sulfide $\left(\mathrm{Sb}_{2} \mathrm{~S}_{3}\right)$ thin films by pulse electrodeposition: Effect of thermal treatment on structural, optical and electrical properties. Materials Science in Semiconductor Processing, 44, 91-100.

[7] Tessler, N. (2016). The band-gap enhanced photovoltaic structure. Applied Physics Letters, 108(18), 183503.

[8] Saito, T. (2012). Optical properties of semiconductor photodiodes/solar cells. Metrologia, 49(2), S118-S123.

[9] Jones, R. O. (2015). Density functional theory: Its origins, rise to prominence, and future. Reviews of Modern Physics, 87(3), 897-923.

[10] Wang, L., Kutana, A. \& Yakobson, B. I. (2014). Many-body and spin-orbit effects on direct-indirect band gap transition of strained monolayer $\mathrm{MoS}_{2}$ and $\mathrm{WS}_{2}$. Annalen der Physik, 526(9-10), L7-L12.

[11] Fabian, J. (2014). Band structure and spin-orbit coupling engineering in transition-metal dichalcogenides. Annalen der Physik, 526(9-10), A89A91.

[12] Zhang, Z. (2014). Spin-orbit DFT with analytic gradients and applications to heavy element compounds. Theoretical Chemistry Accounts, 133(12), 1588 .

[13] Takeda, T. \& Kubler, J. (1979). Linear augmented plane wave method for self-consistent calculations. Journal of Physics F: Metal Physics, 9(4), 661-672.

[14] Schwarz, K., Blaha, P. \& Trickey, S. B. (2010). Electronic structure of solids with WIEN2k. Molecular Physics, 108(21-23), 3147-3166.

[15] Kyono, A., \& Kimata, M. (2004). Structural variations induced by difference of the inert pair effect in the stibnite-bismuthinite solid solution series (Sb,Bi) ${ }_{2} \mathrm{~S}_{3}$. American Mineralogist, 89(7), 932-940.

[16] Engel, E. \& Vosko, S. H. (1993). Exact exchange-only potentials and the virial relation as microscopic criteria for generalized gradient approximations. Physical Review B, 47(20), 13164-13174.

[17] de la Roza, A. O. \& Luaña, V. (2009). Runwien: a text-based interface for the WIEN package. Computer Physics Communications, 180(5), 800-812.

[18] Dufek, P., Blaha, P. \& Schwarz, K. (1994). Applications of Engel and Voskos generalized gradient approximation in solids. Physical Review B, 50(11), 7279-7283.

[19] Pourghazi, A., \& Dadsetani, M. (2005). Electronic and optical properties of BaTe, BaSe and BaS from first principles. Physica B: Condensed Matter, 370(1-4), 35-45.

[20] Carey, J. J., Allen, J. P., Scanlon, D. O. \& Watson, G. W. (2014). The electronic structure of the antimony chalcogenide series: Prospects for optoelectronic applications. Journal of Solid State Chemistry, 213, 116125.

[21] Ambrosch-Draxl, C., \& Sofo, J. O. (2006). Linear optical properties of solids within the full-potential linearized augmented planewave method. Computer Physics Communications, 175(1), 1-14.

[22] Wypych, G. (2016). Handbook of fillers: Fourth edition. Elsevier.

[23] Marton, L. (1956). Experiments on low-energy electron scattering and energy losses. Reviews of Modern Physics, 28(3), 172-183. 